Research Article

\title{
Hidden Multistability in a Memristor-Based Cellular Neural Network
}

\author{
Birong Xu ${ }^{1},{ }^{1}$ Hairong Lin, ${ }^{2}$ and Guangyi Wang ${ }^{3}$ \\ ${ }^{1}$ College of Mechanic and Electronic Engineering, Wuyi University, Wuyishan 354300, China \\ ${ }^{2}$ College of Computer Science and Electronic Engineering, Hunan University, Changsha 410082, China \\ ${ }^{3}$ Institute of Modern Circuits and Intelligent Information, Hangzhou Dianzi University, Hangzhou 310018, China
}

Correspondence should be addressed to Birong Xu; xubirong1@163.com

Received 18 July 2020; Revised 8 September 2020; Accepted 24 September 2020; Published 27 October 2020

Academic Editor: Zhi-Yuan Sun

Copyright (c) 2020 Birong Xu et al. This is an open access article distributed under the Creative Commons Attribution License, which permits unrestricted use, distribution, and reproduction in any medium, provided the original work is properly cited.

In this paper, we report a novel memristor-based cellular neural network (CNN) without equilibrium points. Dynamical behaviors of the memristor-based CNN are investigated by simulation analysis. The results indicate that the system owns complicated nonlinear phenomena, such as hidden attractors, coexisting attractors, and initial boosting behaviors of position and amplitude. Furthermore, both heterogeneous multistability and homogenous multistability are found in the CNN. Finally, Multisim circuit simulations are performed to prove the chaotic characteristics and multistability of the system.

\section{Introduction}

The cellular neural network (CNN) was proposed by Chua and Yang in 1988 for processing signals in real time, which is constituted of an array of the basic circuit units called cells [1]. The CNN has received widespread attention in academia because of its extensive applications, such as image processing, parallel computation, and complicated optimization problems $[2,3]$. Chua and Yang used $\mathrm{CNN}$ to realize image processing and pattern recognition [2]. For solving the constrained optimization problem, Shen et al. designed the utility-based radio resource scheduler by employing CNN [3]. Ref. [4] proposed a random weight change (RWC) algorithm to construct $\mathrm{CNN}$ weight and to make the hardware-based learning on CNN templates feasible. Based on a fuzzy cellular neural network (FCNN), an image encryption method was put forward in Ref. [5].

Ref. [6] further presented a state-controlled cellular neural network (SC-CNN), and a generalized Chua's circuit is constructed by three SC-CNN cells. A Colpitts-like oscillator also is implemented by SC-CNN [7]. An SC-CNN-based circuit could produce strange nonchaotic attractors in Ref. [8].

With the advent of memristors, many types of artificial neural networks have been improved by memristors, such as Cellular neural network (CNN) and Hopfield neural network (HNN) [9-20]. The research on CNN mainly lies in its application [10-12]. A memristor-based CNN was presented in Ref. [9], which could generate chaotic attractors and whose chaotic behaviors were studied. However, a memristor-based CNN without equilibrium points is never proposed. Therefore, a memristor-based $\mathrm{CNN}$ without equilibrium points is introduced in this paper, and its dynamical behaviors are investigated. The memristor-based CNN not only has chaotic features but also shows multistability.

To explore multistability, initial boosting behaviors and attractor coexistence are investigated. Since the memory devices depend on its history, initial boosting behaviors were found in some memristor-based or meminductor-based systems [21-23]. Ref. [21] employed a memristor with sine memductance to construct a memristive jerk system. This novel memristive jerk system had four line equilibrium sets and periodical initial boosting. In Ref. [22], a memristormeminductor system was presented, which produced the amplitude, frequency, and position boosting. These systems have infinitely many equilibrium points. The initial boosting behaviors of a memristor-based system without equilibrium points are not put forward. Hence, we study the initial boosting behaviors in this memristor-based CNN. Moreover, 
multistability is divided into heterogeneous multistability and homogenous multistability [22, 24]. This multistability is heterogeneous multistability if there exist coexisting attractors with different shapes. In contrast, the system owns homogenous multistability if it generates the same shape coexisting attractors with different positions and amplitudes or even frequencies.

This paper is organized as follows: Section 2 proposes the model of a novel memristive neural network. Its nonlinear dynamics is presented in Section 3, while its circuital implementation is completed in Section 4. Finally, conclusions are drawn in the last section.

\section{A Memristor-Based Cellular Neural Network}

The standard SC-CNN cell is defined as [6]

$$
\dot{x}_{i}=-x_{i}+\sum_{k=1}^{n} a_{i k} y_{k}+\sum_{k=1}^{n} s_{i k} x_{k}+i_{k} \text {, }
$$

where $x_{i}$ and $x_{k}$ are the state variables and always are the voltage of capacitors, $y_{k}$ is the output variable, $i_{k}$ is the independent current source, $a_{i k}$ and $s_{i k}$ are the feedback coefficient and state coefficient, respectively. A memristor-based cellular neural network is presented, which consists of one memristor-based CNN cell and two standard SC-CNN cells. The memristor-based CNN cell is shown as Figure 1, from which the state equation is obtained as

$$
\begin{gathered}
\dot{x}_{i}=-M(\varphi) x_{i}+\sum_{k=1}^{n} s_{i k} x_{k}, \\
\dot{\varphi}=x_{i},
\end{gathered}
$$

where a memristor is select as [25]

$$
\begin{gathered}
M(\varphi)=\frac{d q(\varphi)}{d \varphi}=a \varphi^{2}+b \varphi+c, \\
\varphi=v .
\end{gathered}
$$

The memristor-based CNN cell is selected as the first cell, while the second and third ones are the standard SC-CNN cells. The state variables of two standard SC-CNN cells are independent of the output variable, and the state variable of the second cell does not relate to the independent current source. Therefore, the proposed CNN can be expressed as

$$
\begin{gathered}
\dot{x}_{1}=-\left(a \varphi^{2}+b \varphi+c\right) x_{1}+s_{11} x_{1}+s_{12} x_{2}+s_{13} x_{3}, \\
\dot{x}_{2}=-x_{2}+s_{21} x_{1}+s_{22} x_{2}+s_{23} x_{3}, \\
\dot{x}_{3}=-x_{3}+s_{31} x_{1}+s_{32} x_{2}+s_{33} x_{3}+i_{3}, \\
\varphi=x_{1} .
\end{gathered}
$$

In order to better explore the feature of the memristorbased $\mathrm{CNN}$, we let three cells own different numbers of state variables. Set $s_{11}=s_{12}=s_{21}=0$, Equation (4) can be simplified as

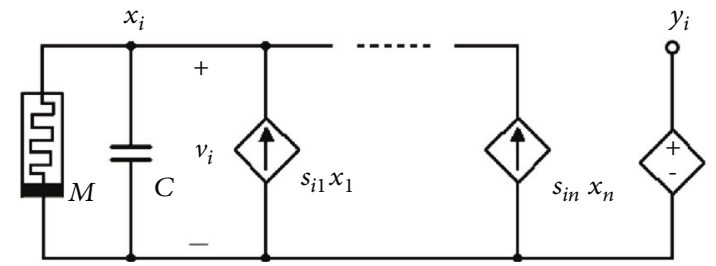

FIgURE 1: A memristor-based CNN cell.

$$
\begin{aligned}
& \dot{x}_{1}=-\left(a \varphi^{2}+b \varphi+c\right) x_{1}+s_{13} x_{32}, \\
& x_{2}=-x_{2}+s_{22} x_{2}+s_{23} x_{3}, \\
& x_{3}=-x_{3}+s_{31} x_{1}+s_{32} x_{2}+s_{33} x_{3}+i_{3}, \\
& \varphi=x_{1} .
\end{aligned}
$$

Obviously, if $i_{3}=0$, the equilibrium point of the $\mathrm{CNN}$ is a line equilibrium set $O(0,0,0, \varphi)$. When $s_{11}=s_{12}=s_{21}=0$, $s_{13}=7, s_{22}=1.75, s_{23}=-1.1, s_{31}=-1.3, s_{32}=1.1, s_{33}=0.85$, $a=20, b=-10, c=-6$, and the initial condition is $(0.1,0$, $0.1,0.3)$, the eigenvalues are $\lambda_{1}=0, \lambda_{2.3}=1.0455 \pm j 1.2192$, and $\lambda_{4}=5.7089$. Thereby, the equilibrium of the CNN is an unstable saddle-focus equilibrium.

If $i_{3} \neq 0$ and $s_{22} \neq 1$, it is easy to see that the neural network is a system without equilibrium. This case will be analyzed below. When $s_{11}=s_{12}=s_{21}=0, s_{13}=7, s_{22}=1.75$, $s_{23}=-1.1, s_{31}=-1.3, s_{32}=1.1, s_{33}=0.85, a=20, b=-10$, $c=-6, i_{3}=-0.0001$, and the initial condition is chosen as $(0.1,0,0.1,0.3)$; the Lyapunov exponent is obtained as LE $1=0.16$, LE2 $=0, \mathrm{LE} 3=0$, and $\mathrm{LE} 4=-27.23$. The CNN is in a chaotic state, whose chaotic attractor and Poincaré mapping are exhibited in Figures 2 and 3, respectively. Since the memristor-based CNN in this case has no equilibrium points, this chaotic attractor is a hidden attractor.

\section{Dynamics of the Memristor-Based Neural Network}

3.1. Influence of the Parameter of the System. In a survey of the dynamical behaviors of the memristor-based CNN, with the parameter $a$ increasing from 9 to 50, and the other parameters and the initial condition set as in Section 2, the Lyapunov exponent spectrum is shown in Figure 4(a), where LE1-3 represent the first three Lyapunov exponents, and the fourth Lyapunov exponent is neglected owing to its large negative value; the corresponding bifurcation diagram is depicted in Figure 4(b). From Figure 4(a), it is easy to observe that the memristor-based CNN keeps a chaotic state. Figure 4(b) shows that the amplitude of $x_{1}$ reduces nonlinearly with the increase of $a$.

3.2. Attractor Coexistence of the Memristor-Based CNN. The memristor-based CNN not only possesses hidden chaotic attractors but also exhibits the phenomenon of attractor coexistence. Setting $s_{11}=s_{12}=s_{21}=0, s_{22}=1.75, s_{23}=-1.1$, $s_{31}=-1.3, s_{32}=1.1, s_{33}=0.85, a=20, b=-10, c=-6$ and $i_{3}$ $=-0.0001$, and varying $s_{13}$, the phenomenon of attractor coexistence is depicted in Figure 5, where the blue orbits start 


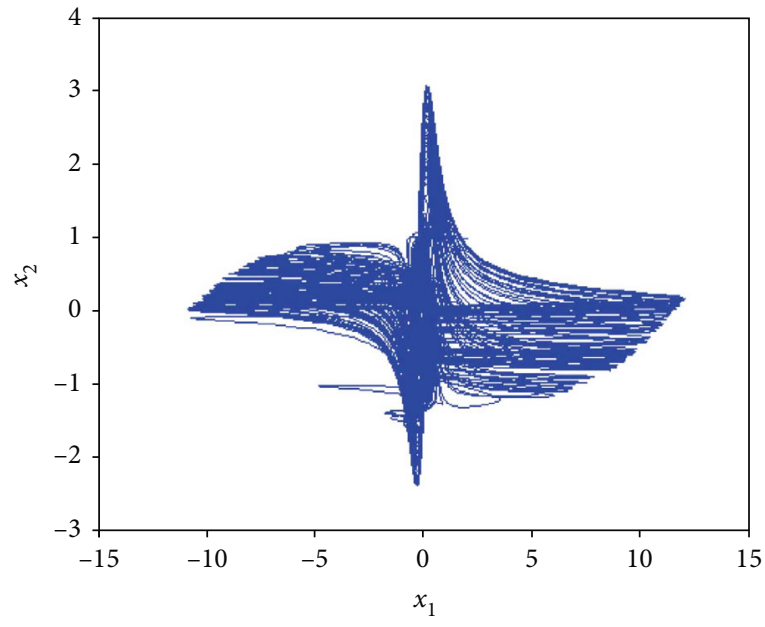

(a)

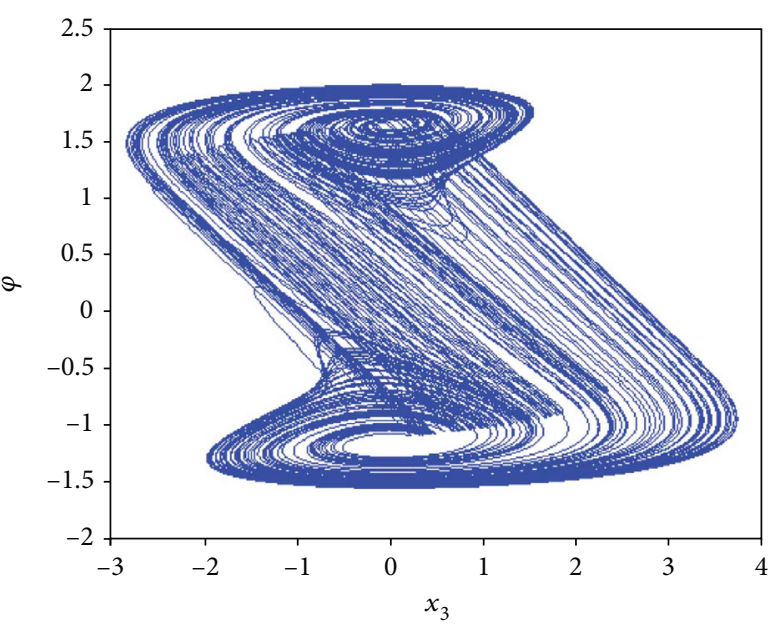

(c)

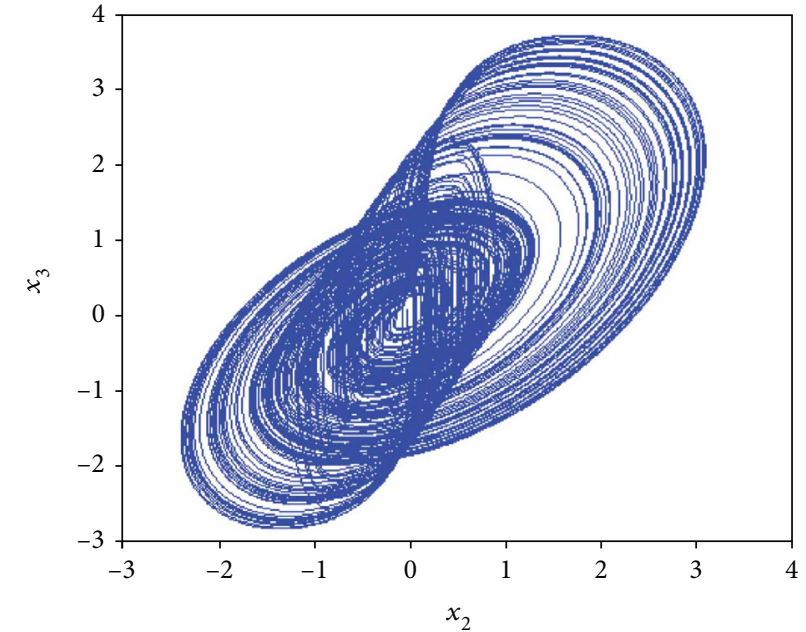

(b)

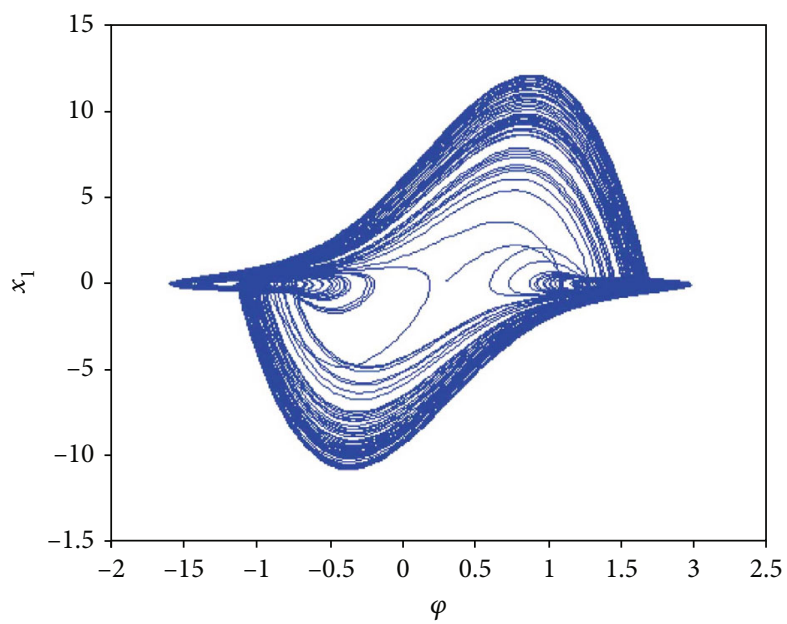

(d)

Figure 2: Chaotic attractors of the memristor-based CNN. (a) $x_{1}-x_{2}$ phase diagram, (b) $x_{2}-x_{3}$ phase diagram, (c) $x_{3}-\varphi$ phase diagram, and (d) $\varphi-x_{1}$ phase diagram.

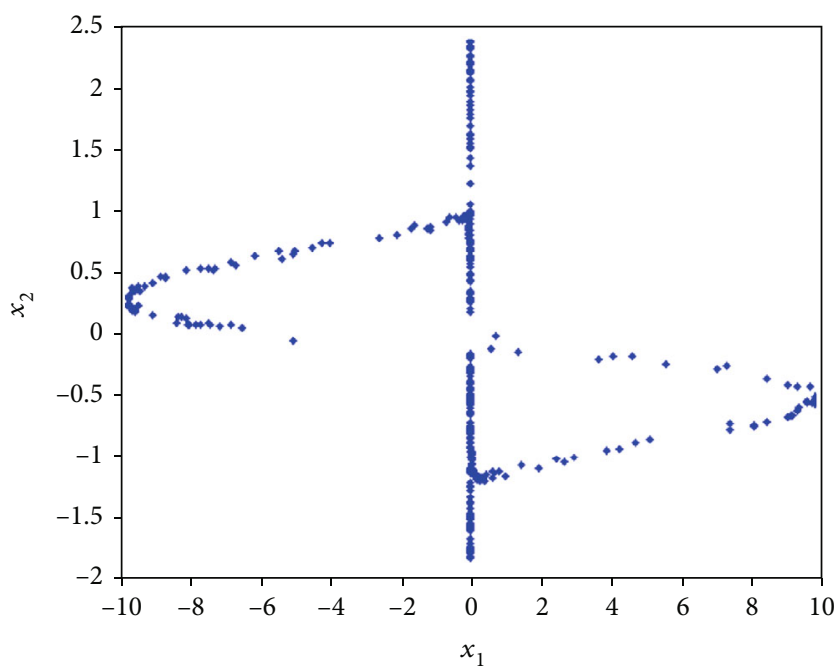

Figure 3: Poincaré map on $x_{3}=0$. 


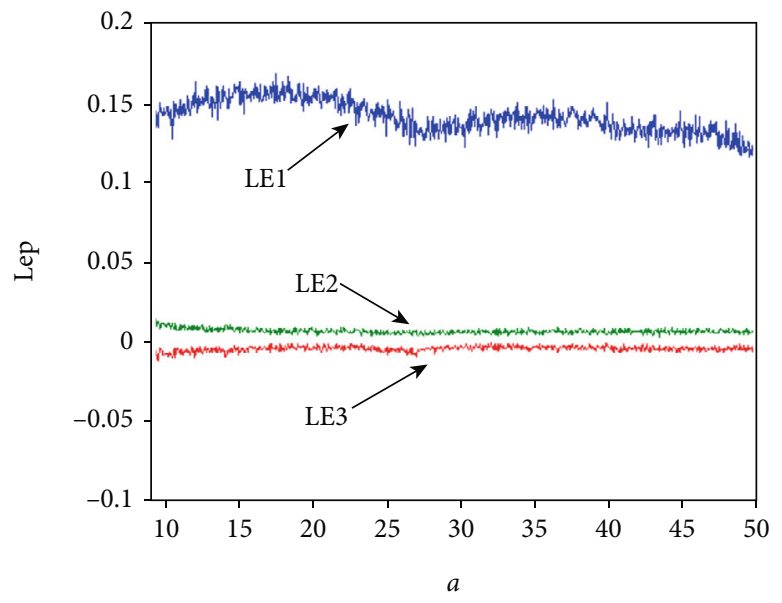

(a)

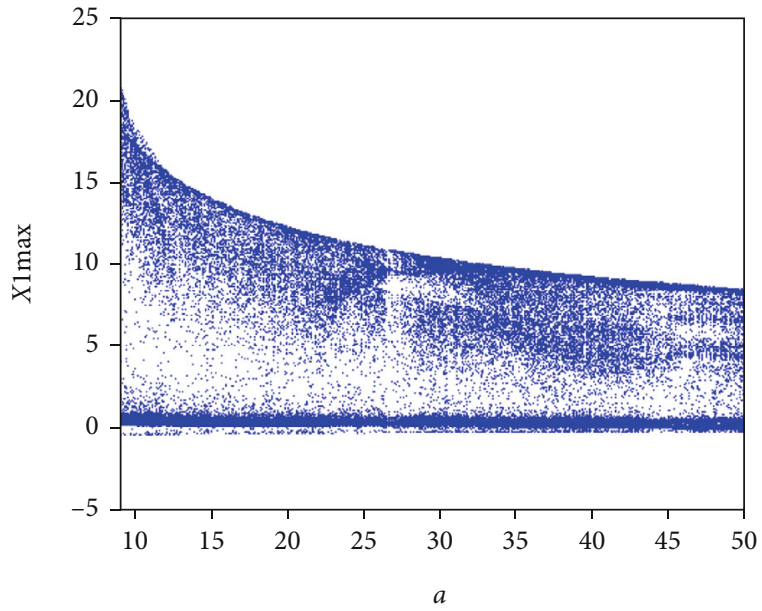

(b)

FIGURE 4: Lyapunov exponent spectrum and bifurcation diagram with respect to $a$ : (a) Lyapunov exponent spectrum and (b) bifurcation diagram.

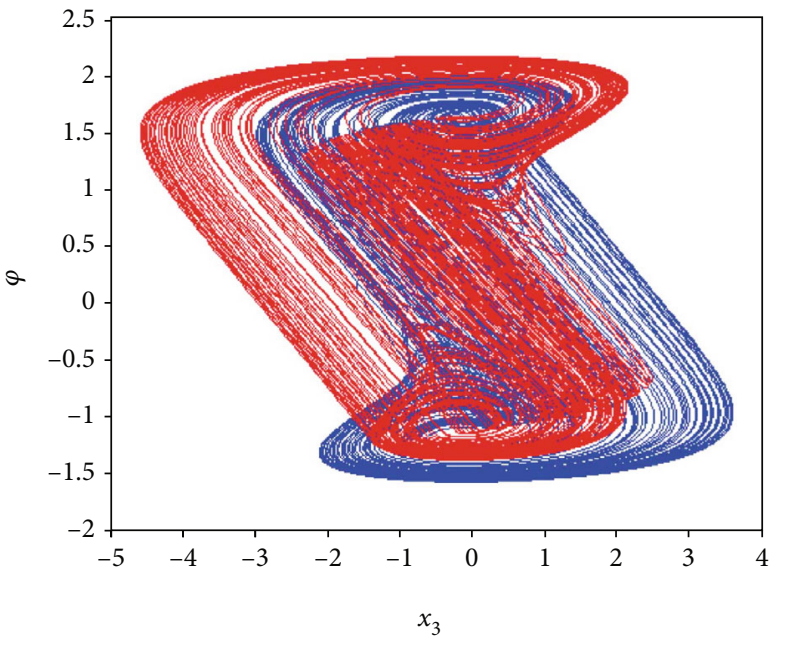

(a)

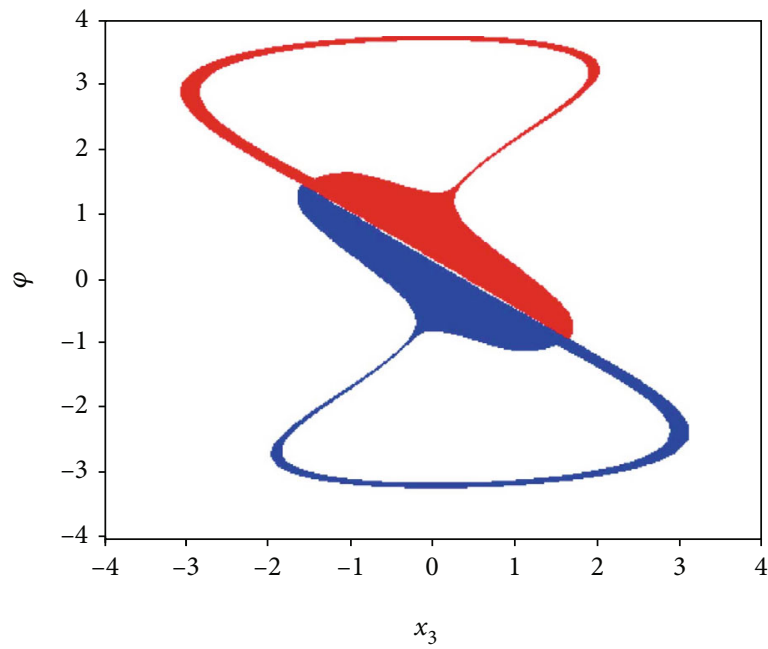

(c)

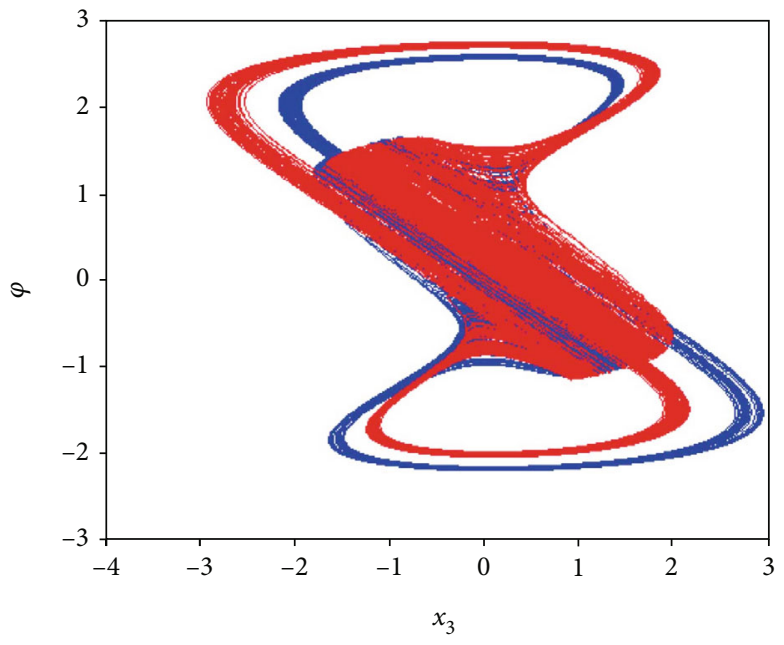

(b)

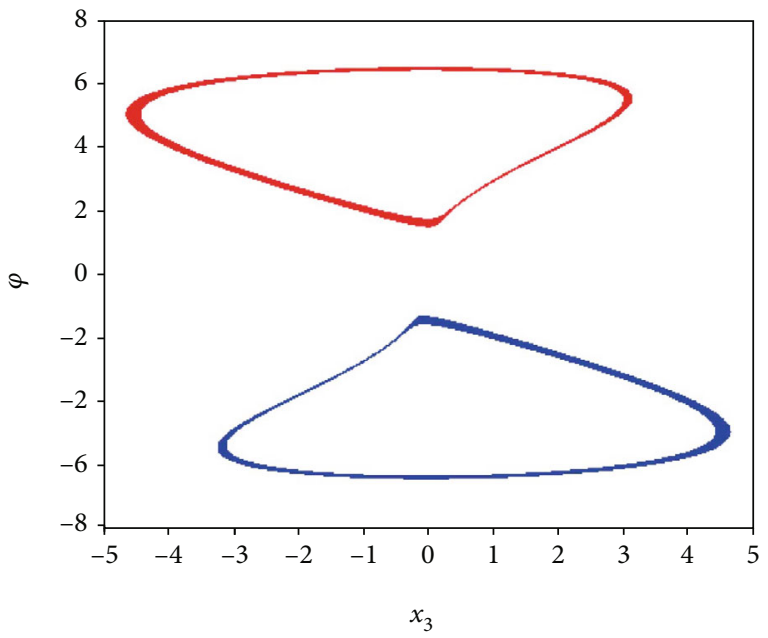

(d)

FIGURE 5: Coexisting attractors on the $x_{3}-\varphi$ plane with different $s_{13}$ under the initial conditions of $(0.1,0,0.1,0.3)(\mathrm{blue})$ and $(0,0,0.3,0)$ (red): (a) coexisting chaotic attractors with $s_{13}=7$, (b) coexisting chaotic attractors with $s_{13}=20$, (c) coexisting periodic attractors with $s_{13}=50$, and (d) coexisting periodic attractors with $s_{13}=200$. 


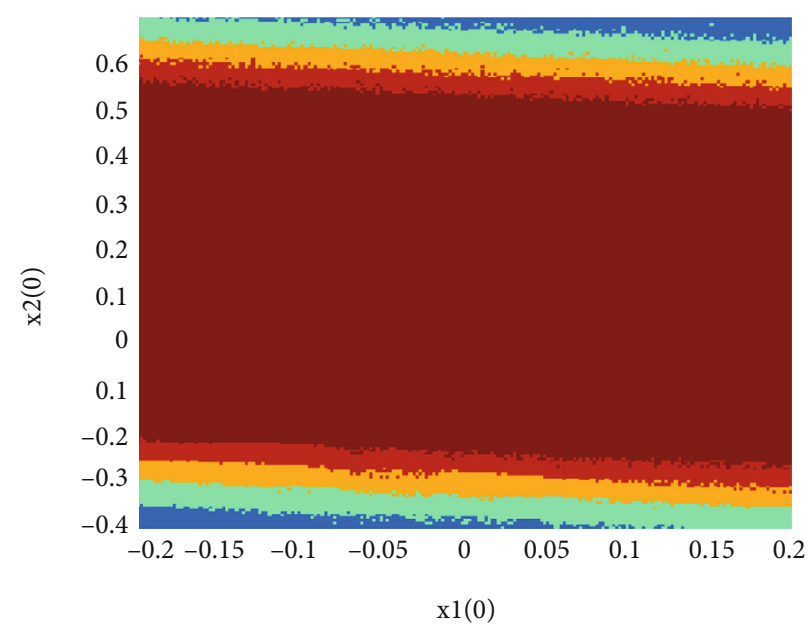

(a)

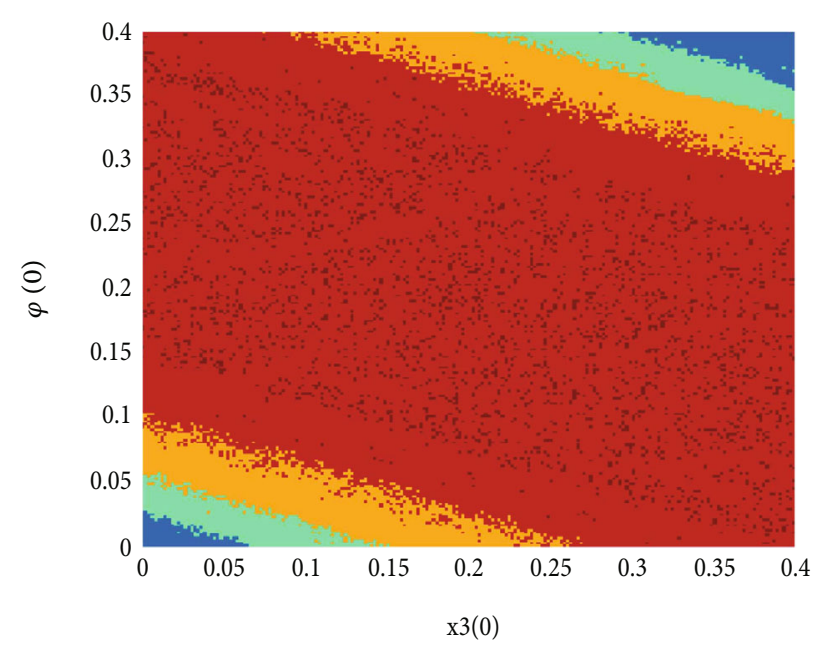

(b)

FIgURe 6: Attractive basins on the cross-section of $s_{11}=s_{12}=s_{21}=0, s_{13}=7, s_{22}=1.75, s_{23}=-1.1, s_{31}=-1.3, s_{32}=1.1, s_{33}=0.85, a=20$, $b=-10, c=-6$, and $i_{3}=-0.0001$ : (a) the attractive basin in the cross-section of $x_{3}(0)=0.1$ and $\varphi(0)=0.3$ and (b) the attractive basin in the cross-section of $x_{1}(0)=0.1$ and $x_{2}(0)=0$.

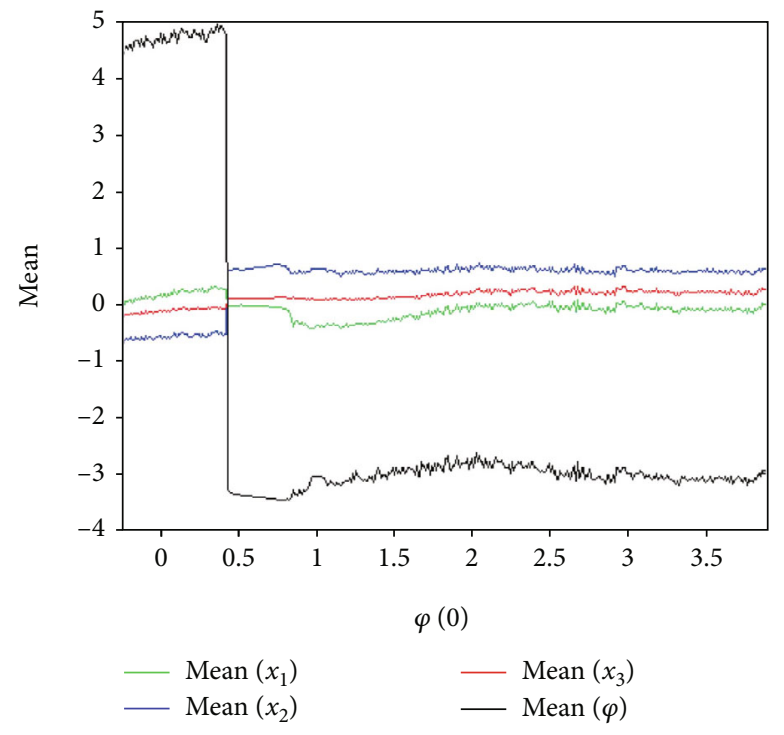

(a)

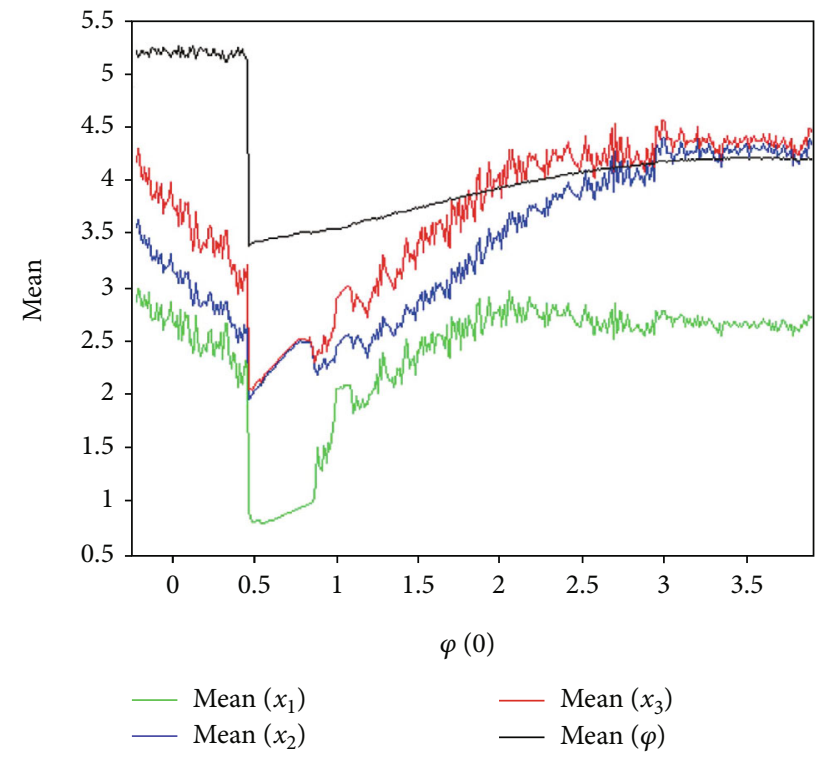

(b)

FIGURE 7: Initial boosting behaviors of position and amplitude with respect to $\varphi(0)$ : (a) initial boosting behaviors of position and (b) initial boosting behaviors of amplitude.

from the initial condition of $(0.1,0,0.1,0.3)$, the red ones from the initial condition of $(0,0,0.3,0)$. The coexisting chaotic attractors are observed at $s_{13}=7$ and 20, whereas the coexisting attractors are periodic at $s_{13}=50$ and 200. The distance of the periodic attractors increases with the increase of $s_{13}$. Moreover, since the memristor-based CNN has coexisting attractors with different shapes, it owns heterogeneous multistability.

Coexisting attractors illustrate that the memristor-based CNN has multistability. To explore its multistability nature, the attractive basins are drawn in Figure 6. Figure 6(a) is the attractive basin in the cross-section of $x_{3}(0)=0.1$ and $\varphi$ $(0)=0.3$, and the other is in the cross-section of $x_{1}(0)=0.1$ and $x_{2}(0)=0$. There are multiple colors in the given value region, which implies several different types of attractors.

3.3. Initial Boosting Behaviors of Position and Amplitude. Initial boosting behavior is a kind of special phenomenon of multistability. Initial boosting behaviors of position and amplitude reveal the attractor's position and amplitude changing with the initial conditions, respectively. When the parameters are chosen as $s_{11}=s_{12}=s_{21}=0, s_{13}=8, s_{22}=1.6$, $s_{23}=-1.1, s_{31}=-1.3, s_{32}=1.1, s_{33}=0.85, a=1.9, b=-3.5$, $c=-6$, and $i_{3}=-0.0001$, the initial boosting behaviors are depicted in Figure 7 , where the initial conditions are $(0,-0.1,0, \varphi(0))$. From Figure $7(a)$, the initial boosting 


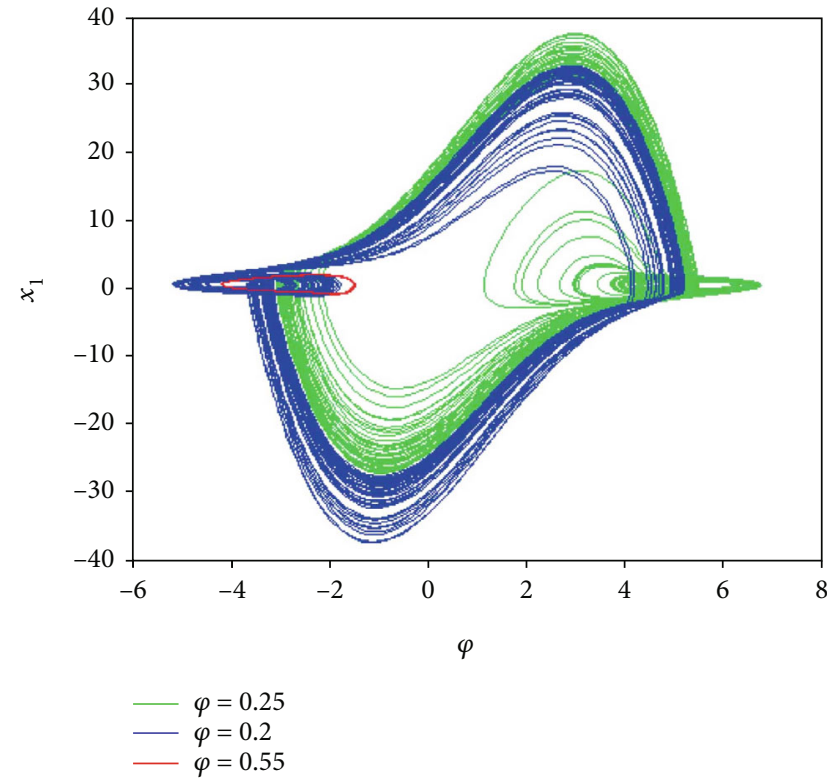

(a)

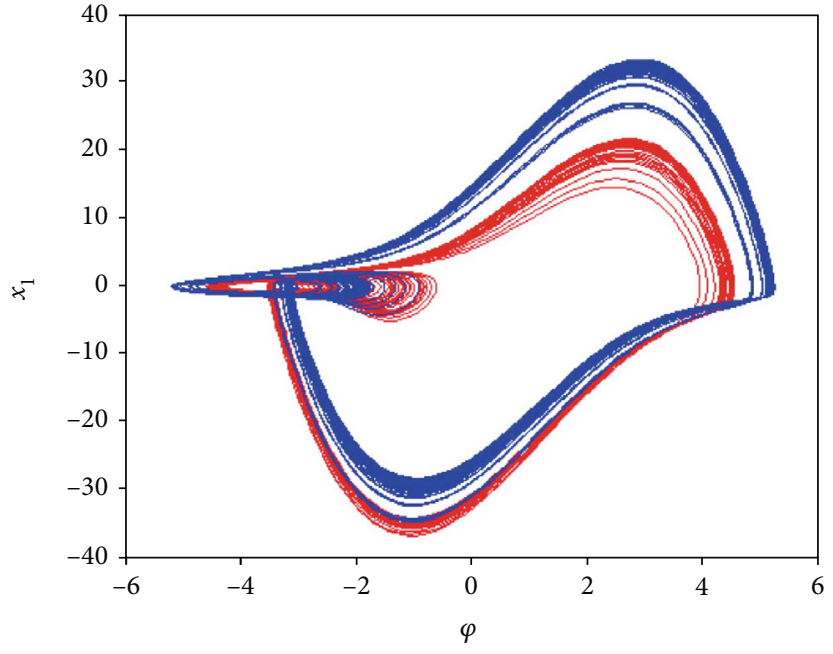

$-\varphi=1.08$

(b)

FIgURE 8: Multistability of the memristor-based CNN: (a) heterogeneous multistability and (b) homogenous multistability.

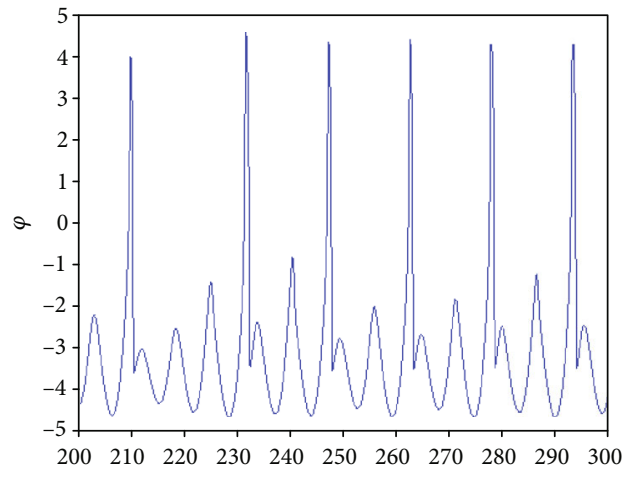

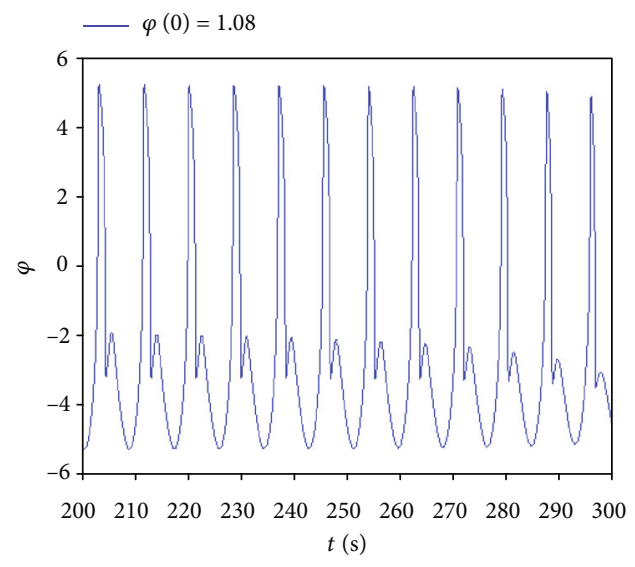

$-\varphi(0)=2.04$

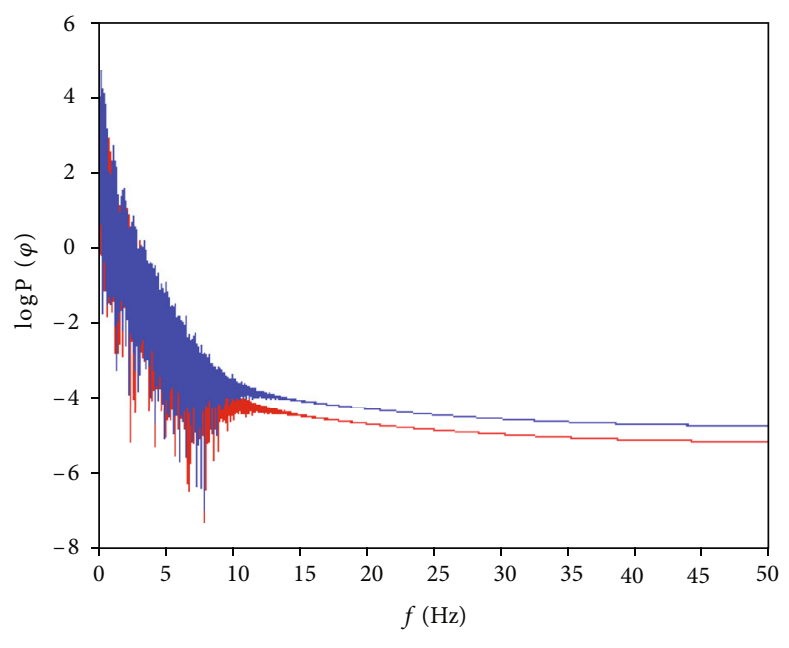

$\varphi(0)=1.08$ $\varphi(0)=2.04$

(a)

(b)

FiguRE 9: Time-domain waveforms and frequency spectra of the chaotic signals with $\varphi(0)=1.08$ and 2.04: (a) time-domain waveforms and (b) frequency spectra.

behaviors of position can be observed. The mean values of the variables $x_{1}$ and $\varphi$ nonlinearly increase, while the other mean values almost do not change, in the range of $\varphi(0) \in[-0.25,0.44]$. When the offset boosting controller is $\varphi(0)=0.44$, there exists a jump for all the mean values. In the region of $\varphi(0) \in(0.44,3.9]$, the mean values of the 


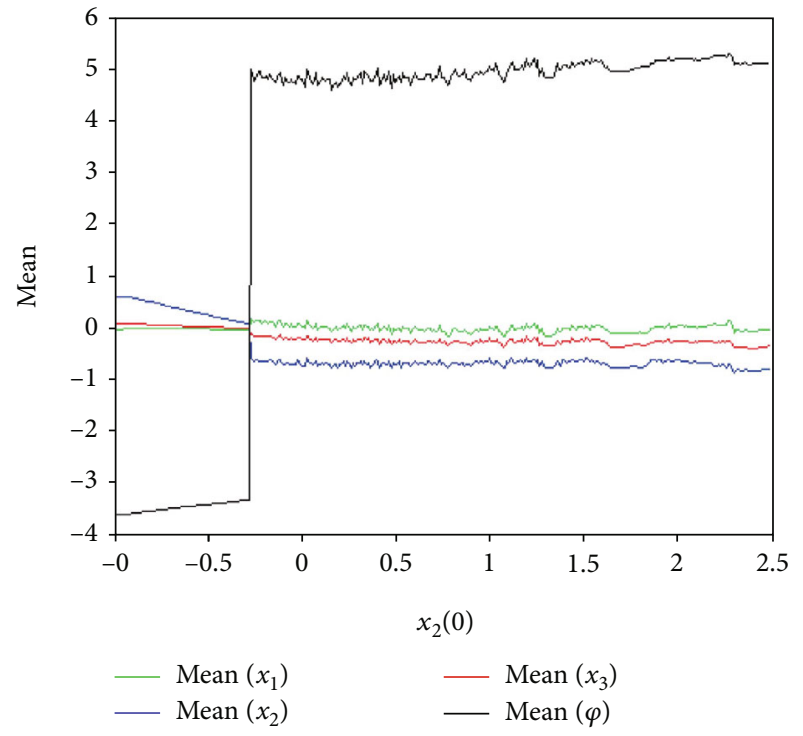

(a)

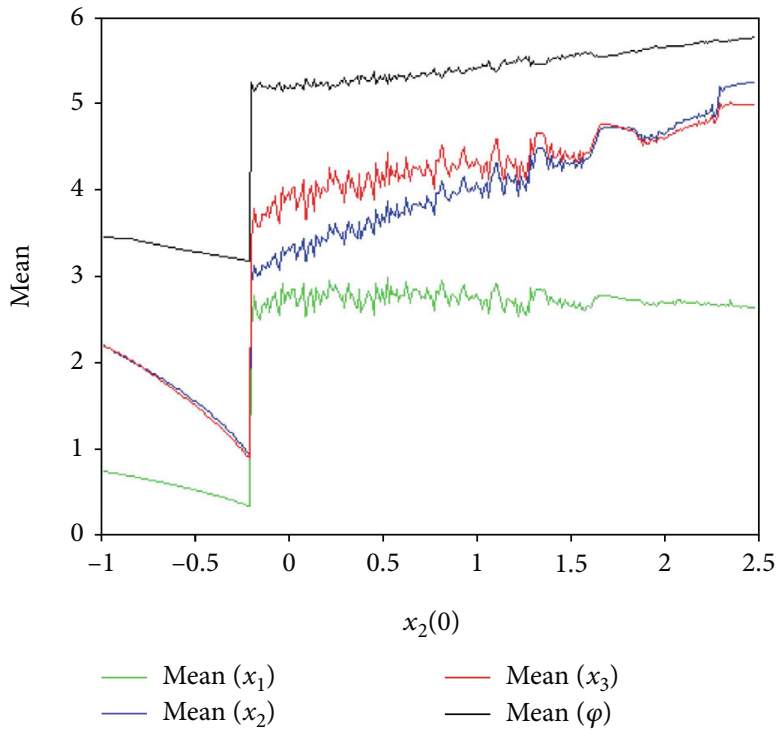

(b)

FIGURE 10: Initial boosting behaviors of position and amplitude with respect to $x_{2}(0)$ : (a) initial boosting behaviors of position induced by $x_{2}(0)$ and (b) initial boosting behaviors of amplitude induced by $x_{2}(0)$.
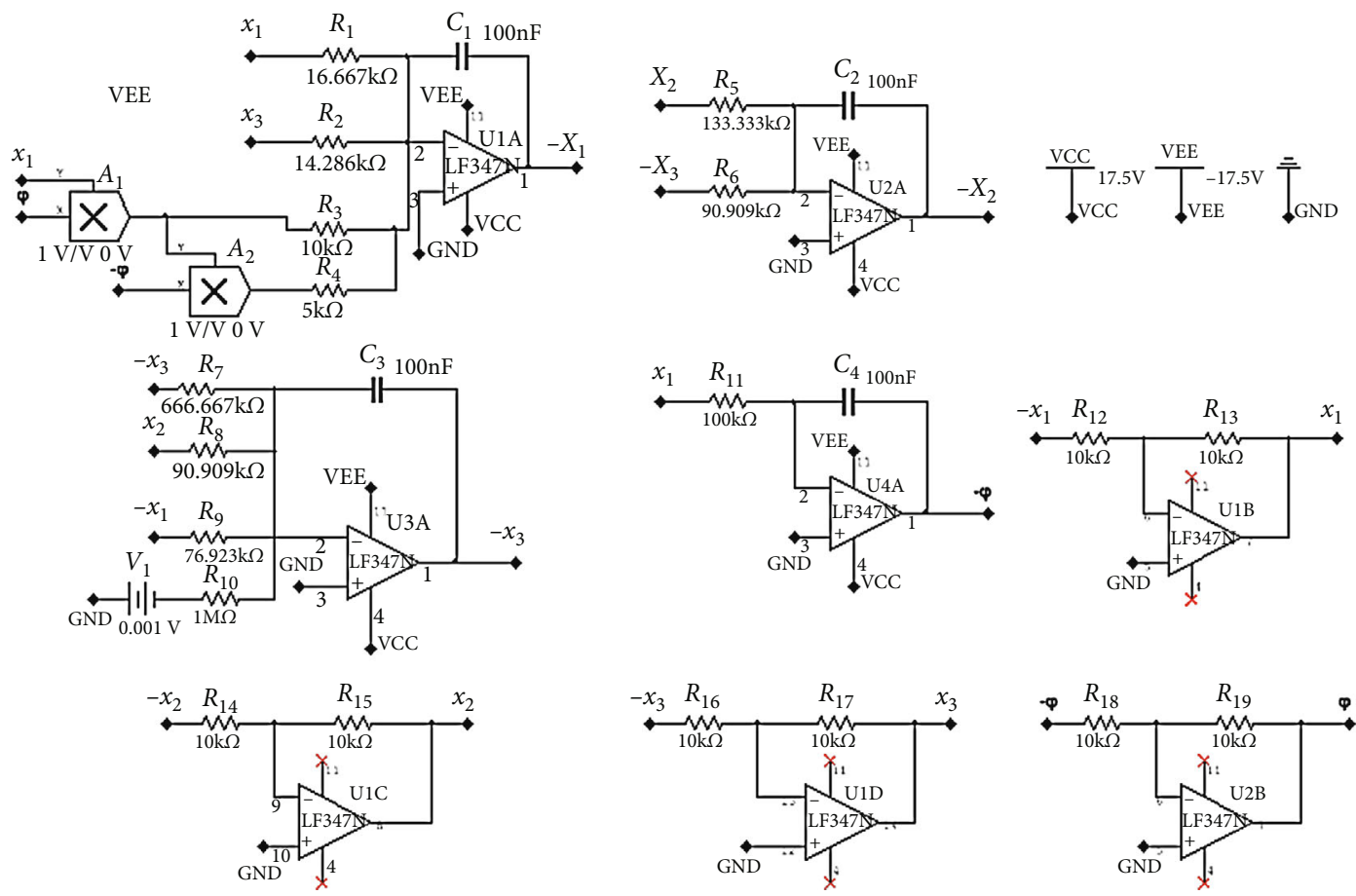

FIgURE 11: Equivalent circuit of the memristor-based CNN without equilibrium points.

variables $x(1)$ and $\varphi$ irregularly change, whereas the mean values of the variables $x_{2}$ and $x_{3}$ still hardly change. Obviously, the route differs from Refs. [21-23].

Moreover, the initial variable $\varphi(0)$ is not only the booster of position but also of amplitude. The initial boosting behaviors of amplitude are shown in Figure $7(\mathrm{~b})$. We can divide the figure into two parts. In the first part $(-0.25,0.44]$, the mean absolute value of the variable $\varphi$ almost keeps unchanged, but the other values nonlinearly decrease; in the second part
$(0.44,2.5]$, all the mean absolute values increase. When the initial value $\varphi(0)$ is 0.44 , all the mean absolute values have a jump, which is the same as the mean values.

For better illustrating the offset boosting, several coexisting attractors are plotted in Figure 8, whose positions and amplitudes are related to the initial variable $\varphi(0)$. From Figure 8(a), three attractors with different shapes are observed, including two chaotic attractors and one periodic attractor, and thus, this system has heterogeneous 


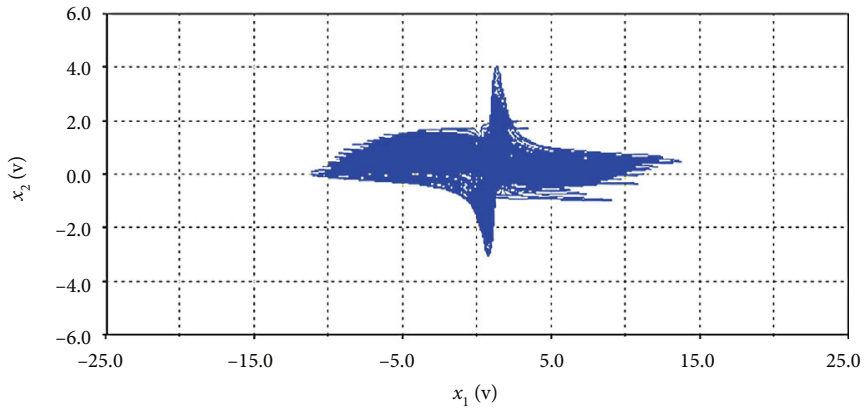

(a)

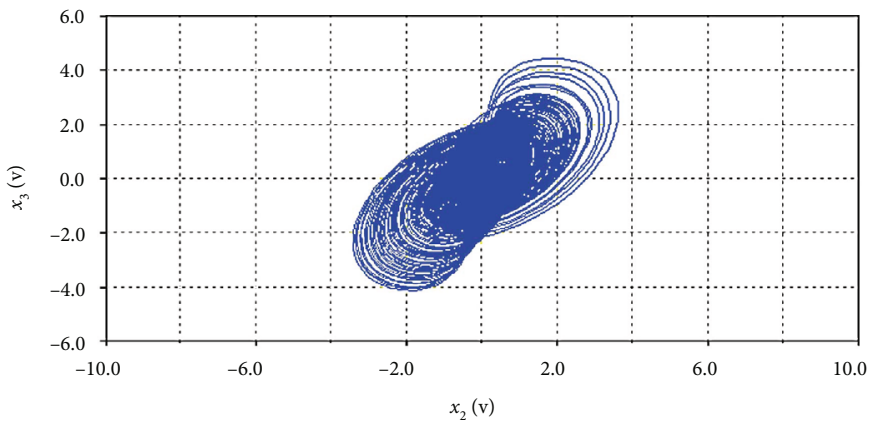

(b)

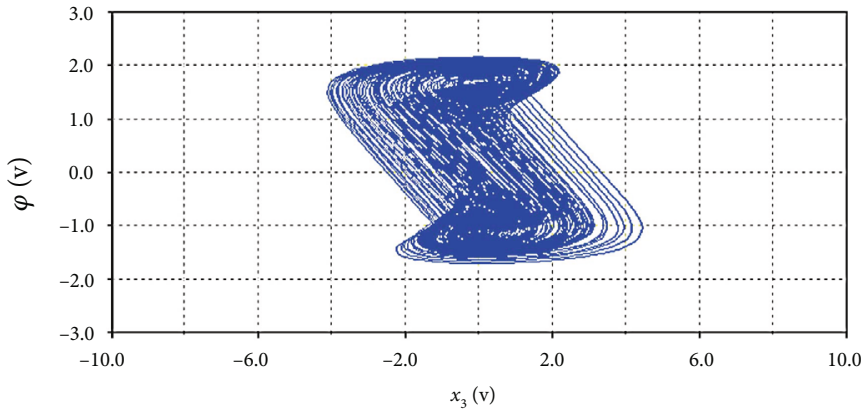

(c)

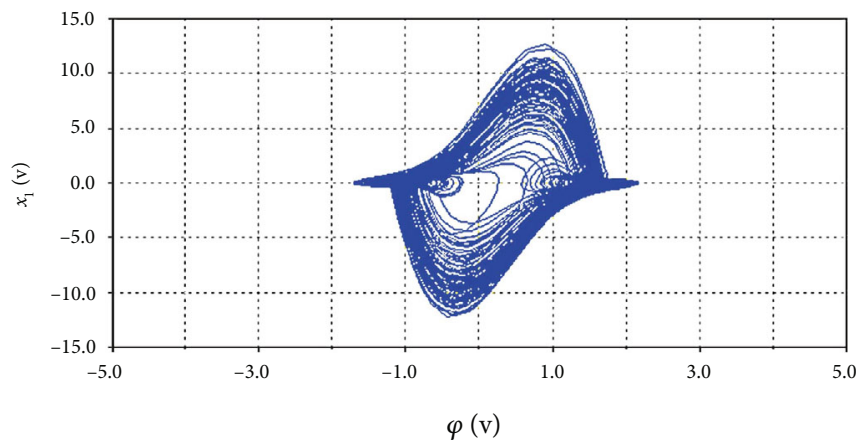

(d)

FIGURE 12: Experimental chaotic orbit: (a) $x_{1}-x_{2}$ phase diagram, (b) $x_{2}-x_{3}$ phase diagram, (c) $x_{3}-\varphi$ phase diagram, and (d) $\varphi$ - $x_{1}$ phase diagram.

multistability. Comparing with Figure 8(a), Figure 8(b) shows the same shape attractors with different positions and amplitudes as shown in Figure 7. Furthermore, if the ODE45 method with the time span $[0,500]$ is used to solve Equation (5), the time-domain waveforms of $\varphi(t)$ are shown in Figure 9(a), illustrating different frequencies with different initial variable $\varphi(0)$. The corresponding frequency spectra of the chaotic signals are depicted in Figure 9(b). Therefore, the multistability is homogenous multistability. This memristor-based CNN owns not only heterogeneous multistability but also homogenous multistability.

The initial variable $\varphi(0)$ is an offset impact factor, but not only. Fixing the parameters, when the initial conditions are set as $\left(0, x_{2}(0), 0,0\right)$, the initial boosting behaviors of position 


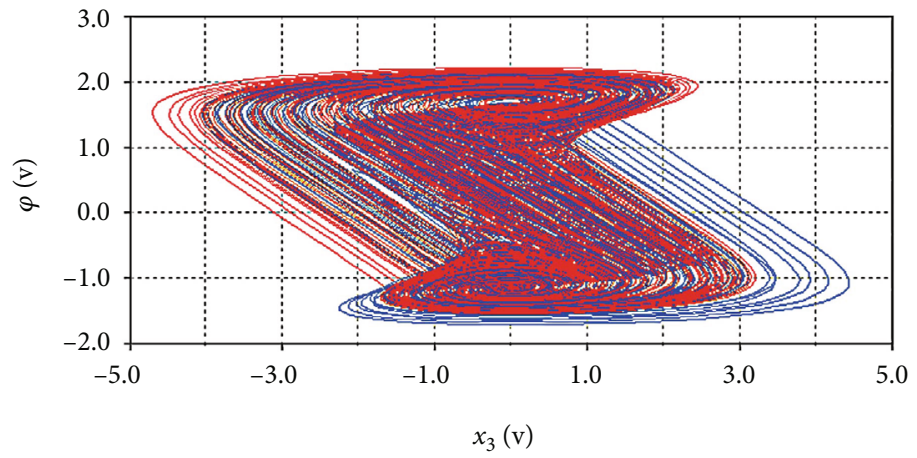

(a)

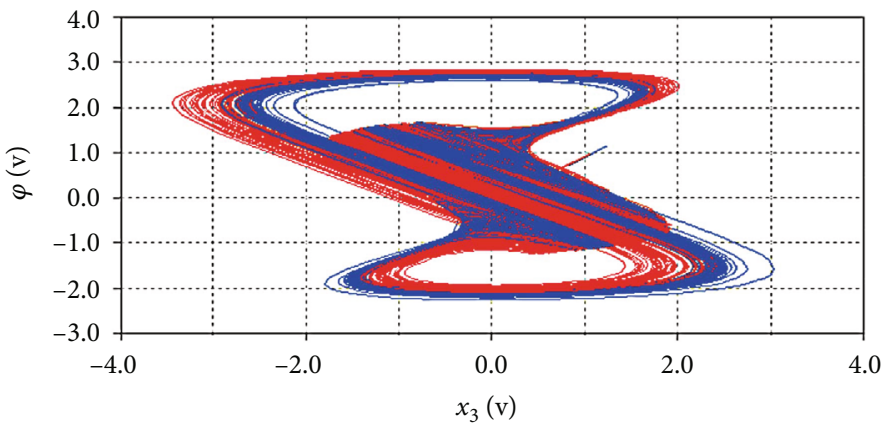

(b)

Figure 13: Experimental coexisting attractors: (a) $R_{2}=14.286 \mathrm{k} \Omega$ and (b) $R_{2}=5 \mathrm{k} \Omega$.

and amplitude are shown in Figure 10. From Figure 10, it is clear to see that the offset adjuster $x_{2}(0)$ also can control the attractor's position and amplitude, but this change process is different from the offset adjuster $\varphi(0)$.

\section{Circuit Design and Experiment Result}

The memristive CNN can be implemented by the circuit. When the parameters are chosen as $s_{11}=s_{12}=s_{21}=0$, $s_{13}=7, s_{22}=1.75, s_{23}=-1.1, s_{31}=-1.3, s_{32}=1.1, s_{33}=0.85$, $a=20, b=-10, c=-6$, and $i_{3}=-0.0001$, and as we introduce the time scale factor $K=100$, the circuit is established as Figure 11. Let $R=100 \mathrm{k} \Omega$, and the state equations are yielded as

$$
\left\{\begin{array}{l}
\dot{x}_{1}=\frac{1}{R C_{1}}\left[-\left(\frac{R}{R_{4}} \varphi^{2}+\frac{R}{R_{3}} \varphi+c\right) x_{1}+\left(\frac{R}{R_{1}}+c\right) x_{1}+\frac{R}{R_{2}} x_{3}\right] \\
\dot{x}_{2}=\frac{1}{R C_{2}}\left[\frac{R}{R_{5}} x_{2}-\frac{R}{R_{6}} x_{3}\right] \\
\dot{x}_{3}=\frac{1}{R C_{3}}\left[\frac{R}{R_{9}} x_{1}+\frac{R}{R_{8}} x_{2}-\frac{R}{R_{7}} x_{1}-\frac{R}{R_{10}} V_{1}\right] \\
\dot{\varphi}=\frac{1}{R_{1} C_{4}} x_{1} .
\end{array}\right.
$$

Employing Multisim to simulate the circuit, the experimental results show that the circuit is in chaos as Figure 12. By giving different initial values and changing the value of the resistor $R_{2}$, the phenomenon of coexisting attractors is obtained as shown in Figure 13. With the resistor $R_{2}=14.286 \mathrm{k} \Omega$, the coexisting attractors in Figure 13(a) are caught by the oscilloscope of Multisim. The red orbit comes from the initial value of $(0.1 \mathrm{~V}, 0$, $0.1 \mathrm{~V}, 0.5 \mathrm{~V})$, while the blue one comes from the initial value of $(0,0,0.1 \mathrm{~V}, 0)$. When the resistor $R_{2}$ is selected as $5 \mathrm{k} \Omega$, Figure 13(b) demonstrates the other phenomenon of attractor coexistence.

\section{Conclusions}

In this paper, we introduce a memristor-based CNN without equilibrium points, which contains a memristor-based CNN cell and two standard SC-CNN cells. By analyzing its dynamical behaviors, the coexisting hidden attractors are found. More interestingly, heterogeneous multistability and homogenous multistability are observed in the CNN. The presented system owns initial boosting behaviors of position and amplitude. Then, the equivalent circuit of the memristor-based $\mathrm{CNN}$ is designed, with which its chaotic and multistable characteristic is verified. Owing to its rich dynamical characteristics, the memristor-based $\mathrm{CNN}$ can be utilized in the information encryption field.

\section{Data Availability}

The data used to support the findings of this study are included within the article. 


\section{Conflicts of Interest}

The authors declare no potential conflicts of interest with respect to the research, authorship, and/or publication of this article.

\section{Acknowledgments}

This work is supported in part by the National Natural Science Foundation of China (Grant Nos. 61771176 and 61271064), the Natural Science Foundation of Fujian Province (Grant No. 2016J01761), and the Natural Science Foundation of Zhejiang Province (Grant No. LY18F010012).

\section{References}

[1] L. O. Chua and L. Yang, "Cellular neural networks: theory," IEEE Transactions on circuits and systems, vol. 35, no. 10, pp. 1257-1272, 1988.

[2] L. O. Chua and L. Yang, "Cellular neural networks: applications," EEE Transactions on Circuits and Systems, vol. 35, no. 10, pp. 1273-1290, 1988.

[3] S. Shen, C. Chang, and L. Wang, "A cellular neural network and utility-based radio resource scheduler for multimedia CDMA communication systems," IEEE transactions on wireless communications, vol. 8, no. 11, pp. 5508-5519, 2009.

[4] S. P. Adhikari, H. Kim, C. Yang, and L. O. Chua, "Building cellular neural network templates with a hardware friendly learning algorithm," Neurocomputing, vol. 312, pp. 276-284, 2018.

[5] K. Ratnavelu, M. Kalpana, P. Balasubramaniam, K. Wong, and P. Raveendran, "Image encryption method based on chaotic fuzzy cellular neural networks," Signal Processing, vol. 140, pp. 87-96, 2017.

[6] P. Arena, L. Fortuna, S. Baglio, and G. Manganaro, "Simplified scheme for realisation of Chua oscillator by using SC-CNN cells," Electronics Letters, vol. 31, no. 21, pp. 1794-1795, 1995.

[7] P. Arena, S. Baglio, L. Fortuna, and G. Manganaro, "How state controlled CNN cells generate the dynamics of the Colpittslike oscillator," IEEE Transactions on Circuits and Systems I: Fundamental Theory and Applications, vol. 43, no. 7, pp. 602-605, 1996.

[8] P. M. Ezhilarasu, M. Inbavalli, K. Murali, and K. Thamilmaran, "Strange non-chaotic attractors in a state controlled-cellular neural network-based quasiperiodically forced MLC circuit," Pramana - Journal of Physics, vol. 91, no. $1,2018$.

[9] Z. Li, Y. Zeng, and Z. Li, "Memristive chaotic circuit based on modified SC-CNNs," Acta Physica Sinica, vol. 63, no. 1, article 010502, 2014.

[10] S. Duan, X. Hu, Z. Dong, L. Wang, and P. Mazumder, "Memristor-based cellular nonlinear/neural network: design, analysis, and applications," IEEE transactions on neural networks and learning systems, vol. 26, no. 6, pp. 12021213, 2015.

[11] S. Duan, X. Hu, L. Wang, S. Gao, and C. Li, "Hybrid memristor/RTD structure-based cellular neural networks with applications in image processing," Neural Computing and Applications, vol. 25, no. 2, pp. 291-296, 2014.

[12] X. Hu, G. Feng, S. Duan, and L. Liu, "A memristive multilayer cellular neural network with applications to image processing,"
IEEE transactions on neural networks and learning systems, vol. 28, no. 8, pp. 1889-1901, 2017.

[13] M. Zheng, L. Li, H. Peng et al., "Finite-time stability and synchronization of memristor-based fractional-order fuzzy cellular neural networks," Communications in Nonlinear Science and Numerical Simulation, vol. 59, pp. 272-291, 2018.

[14] C. Chen, J. Chen, H. Bao, M. Chen, and B. Bao, "Coexisting multi-stable patterns in memristor synapse-coupled Hopfield neural network with two neurons," Nonlinear Dynamics, vol. 95, no. 4, pp. 3385-3399, 2019.

[15] F. Parastesh, S. Jafari, H. Azarnoush, B. Hatef, H. Namazi, and D. Dudkowski, "Chimera in a network of memristor-based Hopfield neural network," The European Physical Journal Special Topics, vol. 228, no. 10, pp. 2023-2033, 2019.

[16] J. Yang, L. Wang, Y. Wang, and T. Guo, “A novel memristive Hopfield neural network with application in associative memory," Neurocomputing, vol. 227, pp. 142-148, 2017.

[17] A. Wu, J. Zhang, and Z. Zeng, "Dynamic behaviors of a class of memristor-based Hopfield networks," Physics Letters A, vol. 375, no. 15, pp. 1661-1665, 2011.

[18] H. Lin and C. Wang, "Influences of electromagnetic radiation distribution on chaotic dynamics of a neural network," Applied Mathematics and Computation, vol. 369, article 124840, 2020.

[19] H. Lin, C. Wang, W. Yao, and Y. Tan, "Chaotic dynamics in a neural network with different types of external stimuli," Communications in Nonlinear Science and Numerical Simulation, vol. 90, article 105390, 2020.

[20] H. Lin, C. Wang, Y. Tan, and Y. Tan, "Hidden extreme multistability with hyperchaos and transient chaos in a Hopfield neural network affected by electromagnetic radiation," Nonlinear Dynamics, vol. 99, no. 3, pp. 2369-2386, 2020.

[21] H. Wu, Y. Ye, M. Chen, Q. Xu, and B. Bao, "Periodically switched memristor initial boosting behaviors in memristive hypogenetic jerk system," IEEE Access, vol. 7, pp. 145022145029, 2019.

[22] F. Yuan, Y. Deng, Y. Li, and G. Wang, "The amplitude, frequency and parameter space boosting in a memristor meminductor-based circuit," Nonlinear Dynamics, vol. 96, no. 1, pp. 389-405, 2019.

[23] H. Wu, Y. Ye, B. Bao, M. Chen, and Q. Xu, "Memristor initial boosting behaviors in a two-memristor-based hyperchaotic system," Chaos, Solitons \& Fractals, vol. 121, pp. 178-185, 2019.

[24] C. Li, W. Joo-Chen Thio, H. Ho-Ching Iu, and T. Lu, "A memristive chaotic oscillator with increasing amplitude and frequency," IEEE Access, vol. 6, pp. 12945-12950, 2018.

[25] W. Liu, F. Q. Wang, and X. K. Ma, "A unified cubic fluxcontrolled memristor: theoretical analysis, simulation and circuit experiment," International Journal of Numerical Modelling: Electronic Networks, Devices and Fields, vol. 28, no. 3, pp. 335-345, 2015. 